\title{
The Critical Lede: New Media and Ecological Balance
}

\author{
W. Benjamin Myers \& Desireé Rowe
}

As co-hosts of the podcast The Critical Lede (TCL), we find ourselves at a moment where new media projects are bursting onto the scene of not only communication/ performance studies but also all fields of academe. That we find ourselves in the midst of this explosion calls for reflexivity on the potentials that new media projects open as well as problematics that might ensue as we sort out how to keep our traditional print/stage heritage vibrant while simultaneously making room for scholarship that works outside of this framework.

The only thing we don't want to do is take ourselves too seriously.

One thing is for sure, though; if it isn't doing it yet, the introduction of new media will fundamentally change scholarship. The introduction of new technology is "neither additive or subtractive" (Postman 18). Postman, in his book Technopoly, instead articulates how we should think about the changes wrought by new technologies as ecological intrusions. Culture is an ecology resting on careful balances where new technologies shift these balances in unpredictable ways. Much like when a new species is presented to an environment, we are not left with the same environment plus the new species. Instead, a radically new environment exists because we have "reconstituted the conditions of survival" (Postman18). The landscape of performance/critical communication scholarship is radically altered by the very possibility of new media scholarship. And once it is introduced, the genie cannot be put back in the bottle.

The possibilities of new media give us the opportunity to perform at being scholars in ways that are both aesthetic and cultural. Anna Deavere Smith claims that "the real 
magic happens when the word hits your breath" (qtd. in Lahr 92). The magic for us is the cultural performance of academics on display, invested with political capital. It is the addition of voice to scholarship that particularly excites us about TCL, and is a unique trajectory when paired with the new media technology environment. Joseph Roach explains that culture (and we see the scholarly communication community as a culture) has two ways of cultural transmission across time: memory and history. Memory is traditionally passed on through embodiment, custom, habit, and stories. It consists of performative reiterations that are ephemeral. They are not stored. History relies on textual knowledge. It is archived and can be accessed at any time. It is public.

Podcasting about our academic culture might hold the possibility of collapsing these two kinds of cultural transmission. The presence of voice to add that "magic" of cultural performance is fundamentally about spontaneity and is radically ephemeral. We perform as scholars; we embody what it means to be active in our culture. Our performances can be thought of as prepared improvisation. We do not rehearse our podcasts, but we do plenty of homework. This might be a kind of memory mode of cultural transmission due to the ephemeral nature of voice and our embodiment of what it means to be performance studies scholars. While our performances are spontaneous, they are also public and provide a perfect digital record. This is the historical method of cultural transmission. We have an archive section on our website whereby anyone can access any one of our performances at any time. New media provides opportunities for transmission through both memory and history. Never before has it been so easy to publicly broadcast oneself to your colleagues as well as to create a perfect storage space where those "memories" can easily be accessed and contribute to a public history.

And while we know that we can not speak with authority, below are a few comments/predictions on how podcasting (and new media in general) might shift balances and thereby alter the ecology of performance studies/critical communication scholarship and alter both the memories and histories of the discipline.

\section{Immediacy}

Who the hell do they think they are?

We have often had conversations around the concern that other scholars might question the authority of our voice in this academic dialogue. Does the space we have created on TCL come off as if we believe we are "authorities" on the subject matter? Do our scholarly achievements in both print and on the stage grant us the legitimacy to speak about scholarship as we do? Having a voice in the field has historically taken a very different course than it did for us. Scholars have traditionally had to produce impressive bodies of work to find their way into the academic dialogue in highly visible ways. This often takes years to accomplish.

The question of "who the hell do they think they are?" is a pertinent one and is intricately tied to the performative possibilities of new media scholarship. To answer 
the question of "who the hell do they think they are?," we could be anyone. One of the possibilities of new media scholarship is the way that it opens up possibilities to anyone with a good idea and the initiative to follow through. We both teach at a rather small university that is generally not at the forefront of performance scholarship. Our careers are young, and while we are ambitious to publish, our vitaes are not padded with numerous publications. In spite of this, we still find ourselves enmeshed in very important conversations with influential scholars because of the performance space that TCL affords.

This performance space helps offer a kind of immediacy or timeliness rarely found in traditional scholarship. Most of us in the discipline are well aware of how long it takes from the time an essay is written until it is in print. But the unscripted nature of the performance space that TCL creates is one that is immediate. This timeliness is one of the more striking possibilities of new media scholarship. The immediacy with which performance scholars can now engage scholarship is astounding.

\section{Politics}

We can't afford subscriptions to all those journals. Sometimes we have to ask you for a copy.

The performative possibilities of new media scholarship are also inherently political, while traditional print scholarship has become increasingly politically problematic. Striphas explains how journals increasingly alienate scholars and divorce them from their labor. The cause of this alienation lies in the fact that journal publishing (including Taylor \& Francis which print all NCA journals including this one) is big business. Striphas convincingly explains how the journal publishing industry works not for authors or scholars, but instead for the bottom line of the companies that have academic journal printing houses in their portfolios. The closed access of most journal articles certainly does not foster disseminating scholarship and making it public.

We see one of the primary functions of TCL as to help work on the side of scholars. We believe that the discipline is a long way from replacing the printed word and the journal publishing industry as the primary source of scholarly engagement. At TCL we do not seek to replace the written word, but instead to help direct scholars to articles/books that might be useful or interesting. We also seek to provide the information from these sources in a venue that is not tied to capital. One need not subscribe to a performance studies/critical communication journal to have access to the information.

While the podcast is open access and available to anyone with a modem, we are also reflexive of the fact that our work at TCL is not necessarily radical. While we disseminate information through voice, we realize that we are intricately tied to the publishing industry. Discussions on TCL originate from traditional print outlets. We understand that TCL can certainly be thought of as an advertisement for traditional print journal articles that might lead to an increase in consumption of those very journals that are owned by large corporations. Striphas explains how an Informa (which owns Taylor \& Francis) subsidiary has worked with the US Army to 
assess battle readiness. It is these big business connections that cause us to pause and wonder about how subversive a new media project intricately tied to scholarly journals can actually be. Perhaps this point should serve to remind us that while new media scholarship does offer us performative possibilities, it is not a silver bullet to making scholarship ethical.

\section{Public Intellectualism}

How do you get more Facebook fans? Begging.

Our physical location is at a small regional university in South Carolina. We are in a department made up of many components (theater, art, digital media, journalism), so we do not have the benefit of a large department made up of many colleagues and graduate students in our discipline with whom we can discuss the latest news in the field. And while Bérubé mentions, "college teaching is a substantial form of "public intellectual' work," we wanted to engage in the types of conversations that we remembered from graduate school (13). So, on the day our first podcast was made public (April 12, 2009), we inserted ourselves into a larger public discourse about critical communication scholarship. We simultaneously opened ourselves up to a world of criticism and subverted dominant networks of power within the discipline.

Unlike what one of our critics noted, we do take TCL very seriously. We understand that the choices we make in terms of articles and interviews mean something. These choices, at the most basic level, reflect scholarship that is interesting to us. But we all know that there is more to choice than this simplicity. We attempt to focus on scholarship that stands out in a way we think might be valuable to our audience by mediating their engagement with the journal issue that often sits unwrapped on an office shelf for months. And this, we believe, is one of the key performative possibilities of new media. Not just to advertise for print media (which is not our goal), but to advocate for a new reading of scholarship that might get overlooked in the deluge of journals.

To put this more eloquently: new media scholarship does not attempt to fit through the premade doors (or, say, hoops) of networks of power. New media builds the doors and then is the very first to walk through. We really like talking about new scholarship within the discipline, and want our audiences to engage in those conversations. Our discussions are our form of public intellectualism, getting the ideas "out there" in order to help build a more fruitful landscape. For example, for this forum issue we are also putting together a special edition of the podcast where each of the authors will be able to discuss their ideas outside of the page.

\section{Dissemination}

We have no idea where to put The Critical Lede on our CVs.

This leads to our next tenet of public intellectualism, the consistency within the discipline of the generating vs. facilitating binary. Graduate students and junior 
faculty push for years towards scholarship - and rightfully so, for many of us need a certain number of publications before we go up for tenure. We do, however, know the heartbreak of being engaged in a piece of scholarship we are working on, only to be disappointedly presenting it to an empty room at a conference center at NCA. Why is that room empty? All your friends are presenting somewhere else! Generating their own scholarships and talking to half-empty rooms. We have both experienced this more than we would like to discuss, and it helped form another potential for new media.

What if we did both? What if it was not just tenured faculty that engaged and formed the discussion as editors of journals? We attempt to make connections between the articles we discuss. For example, we have noticed that quite a few of the articles we have reviewed have engaged the multifaceted discourses of citizenship, so we attempt to bring these discussions together, to cross-reference them and call attention to the level of intersectionality within our work. We are attempting to perform the role of interviewer and interlocutor, generating ideas and discussion and facilitating the spread of those ideas among our colleagues.

We seek to not only disseminate the information contained in the work we discuss, but we also seek to provide a richer read of scholarship by providing context. Before each podcast we communicate with the author(s) of the piece, soliciting "behind the scenes" information about the history of that particular piece and the scholarly history of the author(s). Our goal is to facilitate a community of scholars and add a bit of humanity to the discourse by adding layers to the reading.

\section{Conclusion}

Are we going to do this forever?

The short answer is no. There will be a time when podcasting, new media, and scholarship moves on in new and interesting directions. When the ecology of the field shifts again, moving slowly (for sure) and gradually in another direction, Ben and Des will move with it. In the mean time, however, we hope to offer another type of discourse about scholarship that our audience can engage. Because it is all about the audience, isn't it? And what if people stop listening? Stop caring? We will still be here, talking. Hoping you just might tune in.

\section{References}

Bérubé, Michael. "Teaching to the Six." Pedagogy: Critical Approaches to Teaching Literature, Language, Composition, and Culture 2.1 (2002): 3-15.

Lahr, John. "Under The Skin.” The New Yorker 28 June 1993: 90-94.

Postman, Neil. Technopoly: The Surrender of Culture to Technology. New York: Knopf, 1993.

Roach, Joseph. "Culture and Performance in the Circum-Atlantic World." Performance Studies. Ed. Erin Striff. New York: Palgrave Macmillan, 2003. 124-36.

Striphas, Ted. "Acknowledged Goods: Cultural Studies and the Politics of Academic Journal Publishing." Communication and Critical/Cultural Studies 7.1 (2010): 3-25. 